\title{
UK Renal Registry 11th Annual Report (December 2008): Chapter 12 Epidemiology of Methicillin Resistant Staphylococcus aureus bacteraemia amongst patients receiving Renal Replacement Therapy in England in 2007
}

\author{
Richard Fluck $^{\mathrm{a}}$, Jennie Wilson ${ }^{\mathrm{b}}$, John Davies ${ }^{\mathrm{b}}$, Ruth Blackburn ${ }^{\mathrm{b}}$, Donal O'Donoghue ${ }^{\mathrm{c}}$, \\ Charles RV Tomson ${ }^{d}$

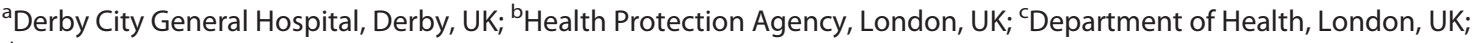 \\ dUK Renal Registry, Southmead Hospital, Bristol, UK
}

\section{Key Words}

Bacteraemia $\cdot$ Dialysis $\cdot$ Vascular access

\begin{abstract}
From April 2007, all centres providing Renal Replacement Therapy in England were asked to provide additional data on patients with Methicillin Resistant Staphylococcus aureus (MRSA) bacteraemia using a secure web-based system. Data were recorded on modality of treatment and the type of vascular access in use at diagnosis and in the previous 28 days. From April 2007 until March 2008, 188 discrete episodes of MRSA bacteraemia were reported in patients receiving dialysis for established renal failure. Over the same period 4,448 MRSA bacteraemias were reported in England, indicating that $4.2 \%$ of all cases occurred in dialysis patients. Of the 188 episodes, additional data from the renal centres were available in 92 cases (49\%). All patients with completed records were
\end{abstract}

\section{KARGER}

Fax +4161306 1234 E-Mail karger@karger.ch www.karger.com
C $2009 \mathrm{~S}$. Karger AG, Basel

$1160-2110 / 09 / 1115-0247 \$ 26.00 / 0$

Accessible online at: www.karger.com/nec on haemodialysis at the time of the bacteraemia. Of those, 65/92 (70.7\%) were using venous catheters, the majority tunnelled lines $(n=55,59.8 \%)$, and 2 other cases had used venous catheters in the previous 28 days. The relative risk of MRSA bacteraemia was about 100 fold higher for a dialysis patient in comparison to the general population and 8 fold higher for a patient using a catheter in comparison to a fistula. The mean rate for all patients was $0.92 \pm 0.85$ episodes $/ 100$ prevalent dialysis patients/year but the rate varied between renal centres with a range of $0-3.28$. Using just haemodialysis patients as the denominator, the mean was $1.14 \pm 0.95$ episodes/100 patients/year with a range of 0-3.93. Compared to previous Registry reports, absolute numbers of reported MRSA bacteraemias has fallen by approximately $62 \%$ from 2004. Many centres have substantially reduced the numbers of cases. Dialysis patients are at increased risk of MRSA bacteraemia; this is closely associated with the use of venous catheters. The rate of MRSA bacteraemia is falling substantially within the prevalent dialysis 
population, but with variation in performance between centres.

\section{Introduction}

Previous analyses have shown that around $8 \%$ of all episodes of MRSA bacteraemia in the UK occurred in patients with established renal failure (ERF) receiving Renal Replacement Therapy (RRT) [1]. The clinical consequences of bacteraemia in patients receiving RRT are well documented [2-7]. There is evidence that the use of catheters for access to the circulation for haemodialysis is associated with increased risk of bacteraemia [8-10] and that an increased risk of bacteraemia may be a major contributor to the higher mortality associated with late presentation with ERF [11].

Previous reports from the UK Renal Registry (UKRR), generated using paper-based survey methods, showed marked variation in provision of vascular access for haemodialysis across the UK, and electronic recording of vascular access provision has now been developed in the UK to support continuing national audit. Dialysisspecific surveillance of bacteraemia has been shown to be feasible within a large UK renal centre [12].

MRSA bacteraemia is a major problem in UK healthcare, and centres providing dialysis contribute a disproportionately high number of cases [13]. Reporting of all MRSA bacteraemia by acute NHS Trusts to the Health Protection Agency has been mandatory in England since 2001, enabling national surveillance $[14,15]$. This report describes the collection of an extended dataset from patients known to have established renal failure.

The term Established Renal Failure used throughout this chapter is synonymous with the terms of End Stage Renal Failure (ESRF) and End Stage Renal Disease (ESRD) which are in more widespread international usage. Within the UK, patient groups have disliked the term 'End Stage' which formerly reflected the inevitable outcome of this disease.

\section{Methods}

All microbiology laboratories in England were required to identify, from the clinical details provided with the sample, all possible instances of MRSA bacteraemia arising in patients undergoing any form of dialysis. Three stages of data completion were required. First, a bacteraemia was identified as being associated with a patient in established renal failure. Second, the record was 'shared' by email alert with the parent renal centre. Third, the renal unit provided additional data on that case, via a web portal.

This process of identification started with completion of a record in the mandatory Healthcare Associated Infection Data Capture System (HCAI-DCS) (previously called Mandatory Enhanced Surveillance System, MESS), an established secure web-based system operated by the Health Protection Agency. The HCAI-DCS collected information on patient identifiers, date the specimen was taken, laboratory where the specimen was processed, the patient's location at the time the sample was taken and whether the patient was an inpatient or outpatient. The system was developed to capture data on whether the patient was on dialysis for acute or established renal failure. When a response indicating that the patient was in ERF was made the user would be prompted to 'share' the record with the renal service. 'Shared' records could be accessed by a designated contact in the renal service who would be informed of a new renal record on the system by an automated email alert. All microbiology laboratories, not just those serving main renal centres or hospitals housing satellite units, were informed about the importance of collection of the extended dataset.

The designated local contact in each renal centre was then required to complete additional fields on each patient with MRSA bacteraemia in ERF via the HCAI-DCS system. This system of data collection was successfully piloted in 8 renal centres prior to inception of the national survey on 1st April 2007. Items collected in these additional fields and the options for completion are summarised in table 12.1 and shown in figure 12.1.

The denominator data used to calculate the rates of MRSA bacteraemia were the numbers of prevalent adult patients receiving haemodialysis or peritoneal dialysis in each centre in the last quarter of 2007, as reported to the UKRR (chapter 4).

\section{Results}

The renal component of the HCAI-DCS went live for all centres in England on 1st April 2007. Data are presented from the first year of collection.

During the period April 2007 until March 2008, a total of 196 MRSA bacteraemias were flagged as being associated with individuals with established renal failure receiving dialysis. Eight of these reports were found to be repeat specimens taken within 48 hours from the same patient (but in different NHS Trusts) during the same episode of bacteraemia and were removed from the analysis, leaving a total of 188 . This represented $4.2 \%$ of the 4,448 MRSA bacteraemia reported in England during this period. Of the 188 episodes, $29(15 \%)$ were not shared with a responsible renal centre, $67(36 \%)$ were shared but not completed 
Table 12.1. Data captured in the HCAI-DCS

\begin{tabular}{|c|c|}
\hline Data item & Options \\
\hline Main renal centre responsible for ongoing care & List of all main renal centres \\
\hline Dialysis centre where the patient receives haemodialysis & List of all dialysis centres affiliated to the main renal centre \\
\hline Modality of dialysis & Unknown/haemodialysis/haemodiafiltration/peritoneal \\
\hline Type of access being used & $\begin{array}{l}\text { Not applicable/unknown/AVF-simple/AVF-complex/AVG/tunnelled venous } \\
\text { catheter J or SC/tunnelled venous catheter - femoral or other/non- } \\
\text { tunnelled venous catheter J or SC/non-tunnelled venous catheter - } \\
\text { femoral or other }\end{array}$ \\
\hline Catheter used in the preceding 28 days & $\begin{array}{l}\text { Unknown/yes/no } \\
\text { If yes, what type? (Unknown/tunnelled venous catheter J or SC/tunnelled } \\
\text { venous catheter - femoral or other/non-tunnelled venous catheter J or } \\
\text { SC/non-tunnelled venous catheter - femoral or other) }\end{array}$ \\
\hline
\end{tabular}

and $92(49 \%)$ had the renal record completed. Table 12.2 summarises the quarterly data for all episodes, including the total number of MRSA bacteraemias reported across England.

During the same period there were 72 episodes reported in patients recorded as being in acute renal failure. These data were derived from the main HCAIDCS reports and are not included in further analysis.
Two episodes of MRSA bacteraemia in children receiving dialysis were recorded, but no information was available from the renal centre in either case. These two cases are not included in the centre-specific analyses.

\section{Access and modality}

For the 92 completed reports, there were no episodes of MRSA bacteraemia recorded for patients on peritoneal

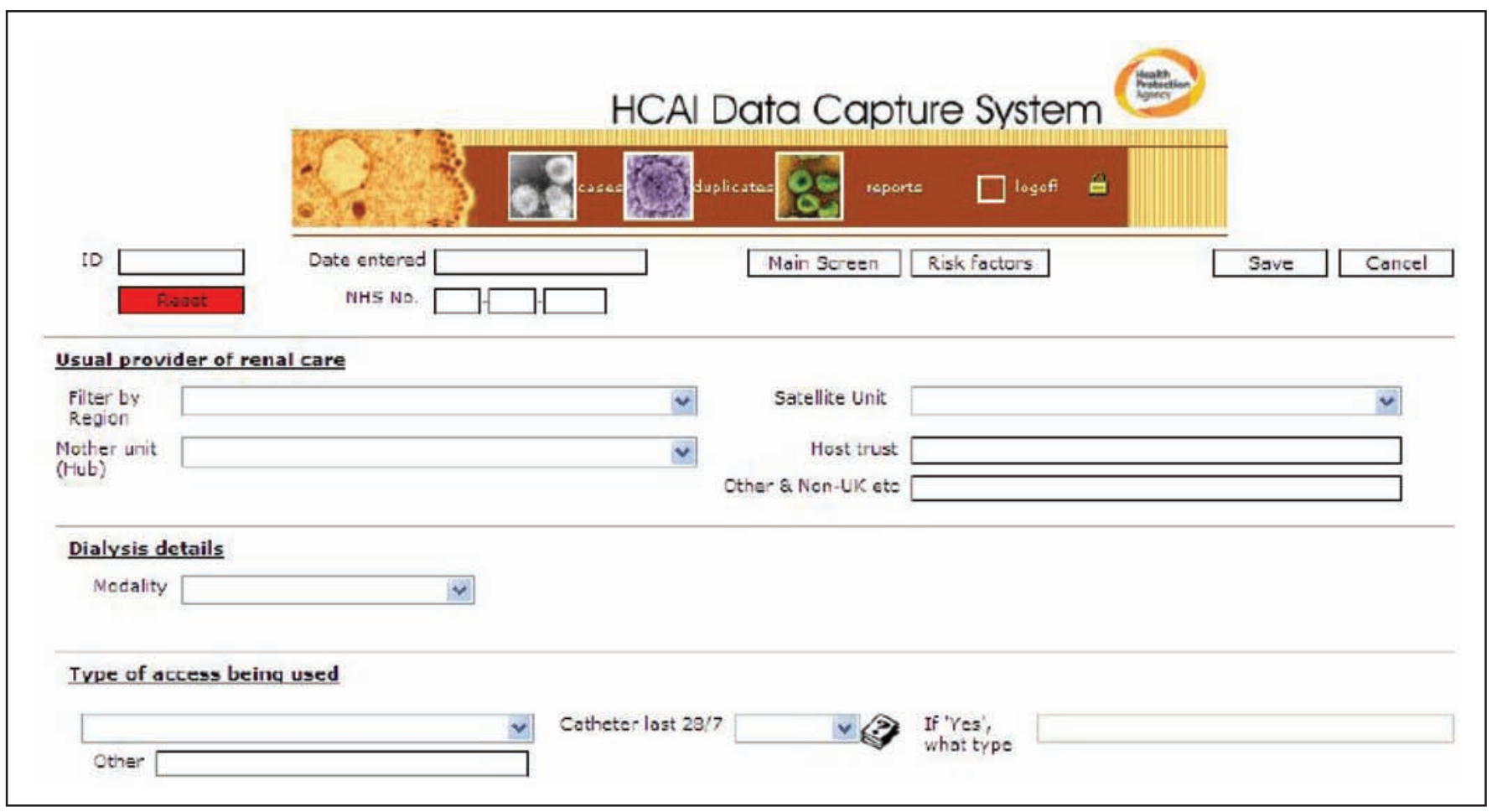

Fig. 12.1. The Renal HCAI-DCS reporting page 
Table 12.2. Number of MRSA bacteraemia and the proportion of records shared with and completed by the renal centre in patients in established renal failure (ERF) reported to the MRSA Healthcare Associated Infection Data Capture System (HCAI-DCS)

\begin{tabular}{|c|c|c|c|}
\hline \multirow[b]{2}{*}{ Period } & \multicolumn{2}{|c|}{ Patients with established renal failure } & \multirow{2}{*}{$\begin{array}{l}\text { All MRSA reported to HCAI-DCS } \\
\qquad \mathrm{N}\end{array}$} \\
\hline & $\mathrm{N}$ & $(\%)$ & \\
\hline April 07-June 07 (26 Trusts) & 59 & $(4.5)$ & 1,306 \\
\hline Not shared & 10 & $(17)$ & \\
\hline Shared, not completed & 17 & $(29)$ & \\
\hline Shared and completed & 32 & $(54)$ & \\
\hline July 07-Sept 07 (29 Trusts) & 44 & $(4.1)$ & 1,082 \\
\hline Not shared & 7 & $(16)$ & \\
\hline Shared, not completed & 14 & (32) & \\
\hline Shared and completed & 23 & $(52)$ & \\
\hline Oct 07-Dec 07 (30 Trusts) & 42 & $(3.8)$ & 1,091 \\
\hline Not shared & 6 & $(14)$ & \\
\hline Shared, not completed & 14 & $(33)$ & \\
\hline Shared and completed & 21 & $(50)$ & \\
\hline Jan 08-Mar 08 (25 Trusts) & 43 & $(4.4)$ & 969 \\
\hline Not shared & 5 & $(12)$ & \\
\hline Shared, not completed & 22 & (51) & \\
\hline Shared and completed & 16 & $(37)$ & \\
\hline April 07-Mar 08 & ${ }^{*} 188$ & $(4.2)$ & 4,448 \\
\hline Not shared & 29 & $(15)$ & \\
\hline Shared, not completed & 67 & (36) & \\
\hline Shared and completed & 92 & (49) & \\
\hline
\end{tabular}

* This excludes 8 records where 2 specimens were taken from same patient with ERF within 48 hrs at different Trusts

dialysis. All patients were on haemodialysis or haemodiafiltration, with 2 subjects where the modality was recorded as unknown (table 12.3).

Table 12.4 details the recorded type of access in use at the time of the episode for the 92 completed renal records. Twenty seven patients $(29.3 \%)$ were using either a fistula $(n=23)$ or graft $(n=4)$. The remainder $(\mathrm{n}=65,70.7 \%)$ were using venous catheters, the majority of which were tunnelled lines $(n=55,59.8 \%)$. Access type was recorded as unknown in one patient.

If a patient was noted as being on $\mathrm{PD}$, or on $\mathrm{HD}$ with a fistula or graft, the use of any venous catheters during the last 28 days was requested. Two patients dialysing on $\mathrm{AV}$ grafts at the time of diagnosis of MRSA bacteraemia had

Table 12.3. Modality of dialysis in patients in established renal failure where record shared and completed

\begin{tabular}{lcc} 
& \multicolumn{2}{c}{ MRSA bacteraemia } \\
\cline { 2 - 3 } Modality of dialysis & $\mathrm{N}$ & $(\%)$ \\
\hline Haemofiltration & 3 & $(3.3)$ \\
Haemodialysis & 87 & $(94.6)$ \\
Unknown & 2 & $(2.2)$ \\
All & 92 & $(100)$ \\
\hline
\end{tabular}

used venous catheters in the prior 28 days. Two patients on $\mathrm{AV}$ fistulae at the time of diagnosis had an unknown status recorded in this data field. Therefore, at least $67 / 92$ $(72.8 \%)$ patients were using or had used venous catheters in the 28 days preceding the MRSA bacteraemia. Data collected during a national paper-based census of dialysis centres during 2004 [1] were used to provide a rough estimate of denominator data: on the assumption that the proportion of patients in the UK using catheters $(23 \%)$ had not changed substantially since that census, and using up-to-date data on total prevalent dialysis

Table 12.4. Type of renal access in patients in established renal failure where record shared and completed

\begin{tabular}{lrr}
\hline & \multicolumn{2}{c}{ MRSA bacteraemia } \\
\cline { 2 - 3 } Renal access type & $\mathrm{N}$ & $(\%)$ \\
\hline AV - simple & 23 & $(25.0)$ \\
AVG & 4 & $(4.4)$ \\
Non-tunnelled - femoral & 46 & $(6.5)$ \\
Non-tunnelled - jugular or subclavian & 4 & $(4.4)$ \\
Tunnelled - femoral & 5 & $(5.4)$ \\
Tunnelled - jugular or subclavian & 50 & $(54.3)$ \\
All & 92 & \\
\hline
\end{tabular}




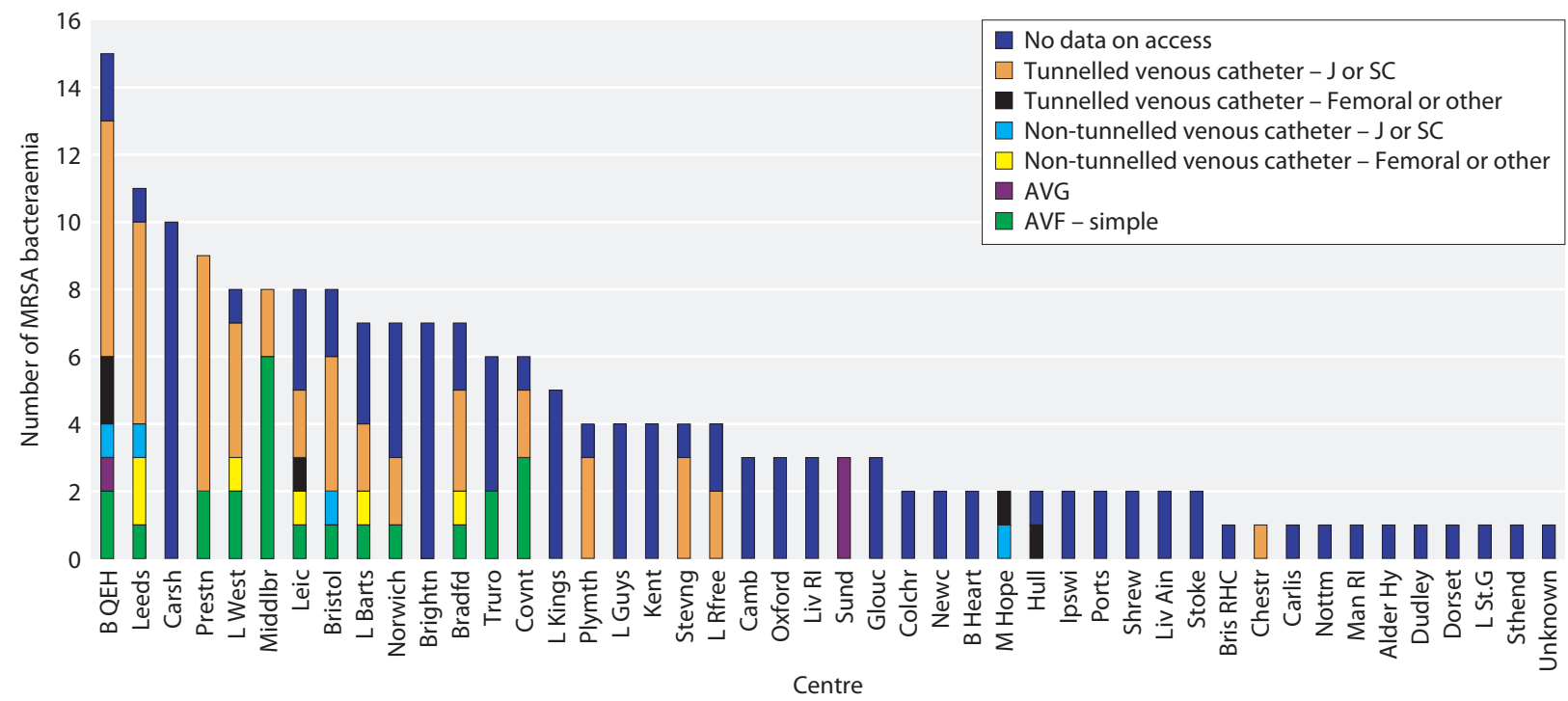

Fig. 12.2. Number of MRSA bacteraemia by access type and renal centre in reporting NHS Trusts (all records $\mathrm{N}=188$ )

patients $(n=20,042)$, this gave a rate of MRSA bacteraemia of 65/4,611 amongst those dialysing using a catheter and 27/15,431 amongst those using a fistula, graft, or peritoneal dialysis. This suggests the relative risk of MRSA bacteraemia was about 8 fold higher for a patient being dialysed on a venous catheter than via a fistula.

\section{Incident episodes by centre}

Fifty acute NHS Trusts reported at least one MRSA bacteraemia in association with a patient with established renal failure on dialysis. Within England, there are 52 distinct renal centres and for the purposes of further analysis, 'unshared' records were allocated to the renal centre thought most likely to be providing long-term supervision of dialysis treatment. The number of MRSA bacteraemia by the type of dialysis access are shown by the renal centre in the reporting NHS Trust (figure 12.2) and for those records where the data was shared with a specific renal centre (figure 12.3).

In calculating the rates of MRSA bacteraemia by renal centre, 3 of the 188 shared records were excluded (two from paediatric units, and one reported by a Trust

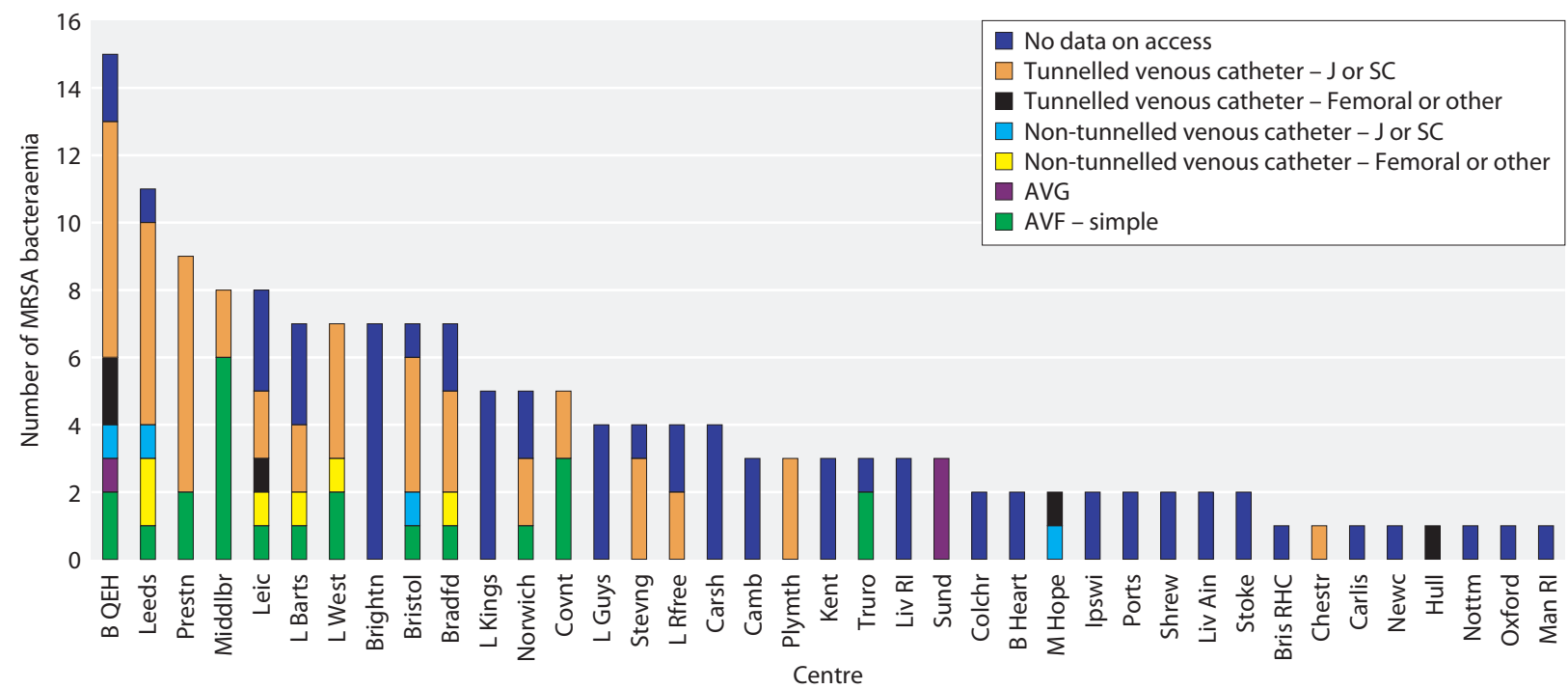

Fig. 12.3. Number of MRSA bacteraemia by renal access type and renal centre (shared records only) $N=159$ 


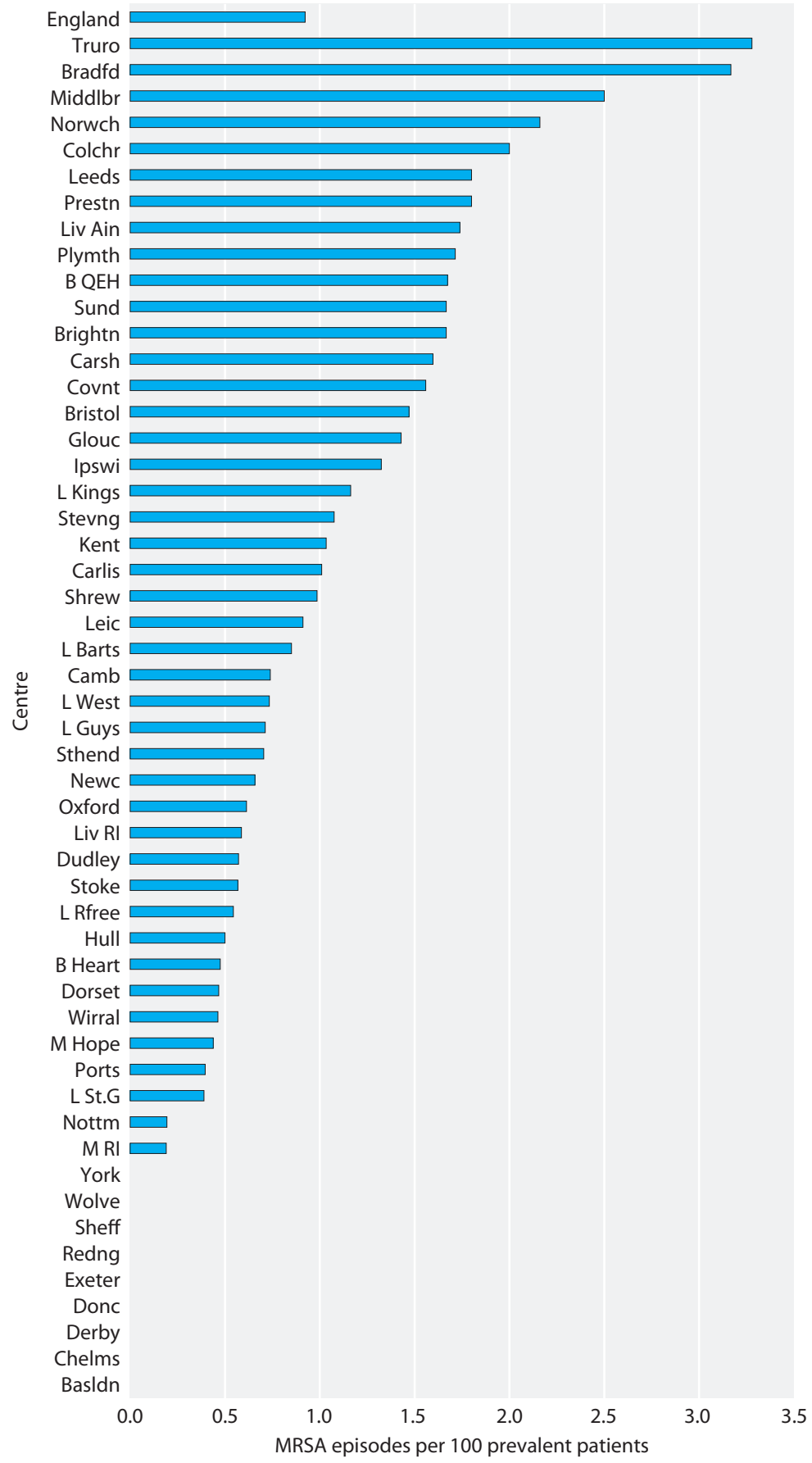

Fig. 12.4. Centre-specific MRSA bacteraemia rates per 100 prevalent dialysis (haemodialysis and peritoneal dialysis) patients equidistant from two renal centres could not be allocated to a renal centre), resulting in a total of 185 records. Nine centres had no episodes during 2007/8.

Figures 12.4 and 12.5 provide relative rates of infection by centre. Figure 12.4 indicates the rates by renal centre per 100 prevalent dialysis patients (PD and HD), and figure 12.5 the rates per $100 \mathrm{HD}$ patients. The mean rate for all patients was $0.92 \pm 0.85$ episodes $/ 100$ dialysis patients, range $0-3.28$. Since all patients with MRSA bacteraemia were on haemodialysis, using just haemodialysis patients as the denominator the mean was $1.14 \pm 0.95$ episodes/100 patients, range 0-3.93.

The proportion of renal records completed on the HCAI-DCS system was disappointingly only $49 \%$. In most cases, centres either reported on all records or did not report on any records. Preliminary investigation 


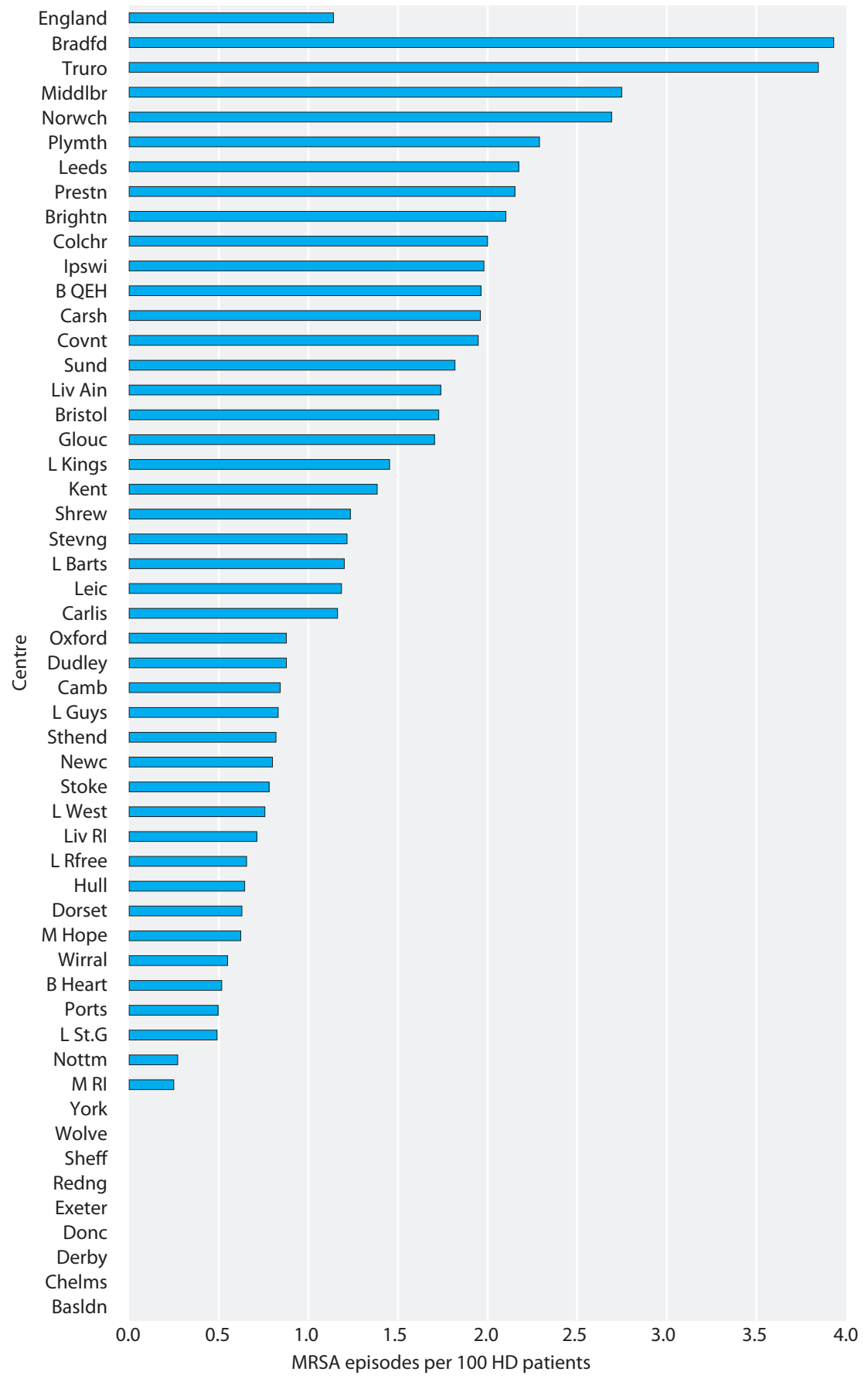

Fig. 12.5. Centre-specific MRSA bacteraemia rate per 100 haemodialysis patients has identified problems with the system for sending an email alert to the designated infection control lead at the renal centre, with errors in the email addresses causing the alerts not to be delivered and in some cases the emails to have not reached the correct inbox. This suggests that the process of sharing and issuing reminders to complete these data fields requires revision.

\section{Comparison with vascular access survey data}

In 2005 the 8th Registry Report produced the results of the National Vascular Access survey, covering the entire United Kingdom. Sixty two centres reported on dialysis access in use in prevalent and incident haemodialysis patients and on Staphylococcus aureus bacteraemia rates 


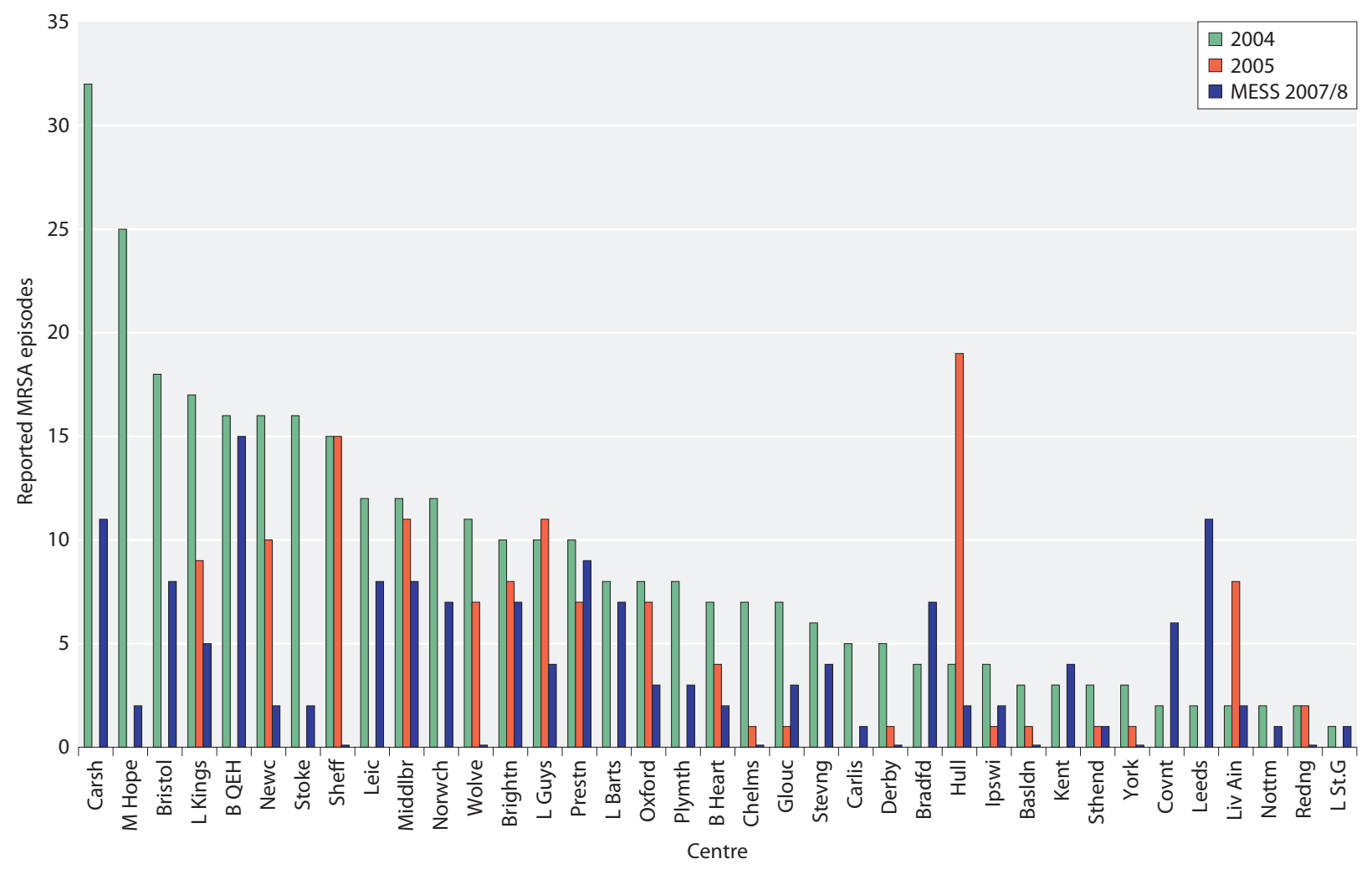

Fig. 12.6. Change in reported MRSA bacteraemia rates in centres that provided data for the previous Registry census

(both total and MRSA) in 2004 [1]. In 2006, 37 centres participated in a follow up survey [13]. While the data were not collected in an identical fashion, comparisons are valuable. In 2005 the 8th Registry Report produced the results of the National Vascular Access survey, covering the entire United Kingdom. Data on MRSA bacteraemias during 2004 were available from 37 English centres, which reported a total of 328 episodes. These centres provided care for 13,644 dialysis patients on 31st December 2004 - giving an overall bacteraemia rate of 2.40 episodes per 100 dialysis patients. This rate was highly likely to be a significant underestimate, given that episodes diagnosed in hospitals other than that housing the renal centre may not have been captured. If these data were representative of England as a whole, given that the current data give an overall rate of 0.92 episodes per 100 dialysis patients, this gives a conservative estimate that there has been a $62 \%$ reduction in MRSA bacteraemia rates amongst dialysis patients in England. This compares favourably with a $42 \%$ national reduction reported by the HCAIDCS surveillance system between 2004 and 2008 [16].

Figure 12.6 breaks down episodes by centre, over the 3 reporting periods, for centres that reported data for 2004. Several centres recorded zero rates in 2007 despite high rates in 2004 - these included Basildon, Chelmsford, Derby, Reading, Sheffield, Wolverhampton and York. Several other centres achieved substantial reductions.

\section{Discussion}

Mortality due to sepsis in patients on dialysis is 100-300 fold higher than in the general population [2]. MRSA bacteraemia contributed markedly to morbidity and mortality in the UK dialysis population, and reducing MRSA bacteraemia specifically amongst dialysis patients has therefore become a priority for policymakers as well as for patients and clinicians.

Within England the MRSA surveillance system has provided data on rates of MRSA bacteraemia for all acute NHS Trusts, allowing for performance in relation to infection control to be tracked and improved. The 2005 Registry Report [1] identified MRSA bacteraemia as an important issue for patients receiving dialysis, for whom the relative risk of this infection was 200 compared to the general population. As a consequence of this analysis, the National Clinical Director for Kidney Care set up a collaboration 
between the Health Protection Agency and the renal community to improve and enhance the reporting of dialysis associated MRSA bacteraemia.

The first year of data collection has demonstrated issues related to producing a complete dataset, and further work is required to improve the system of 'sharing' records once an MRSA bacteraemia has been diagnosed so as to ensure that the requisite information is provided by the renal centre responsible for the patient's care.

Despite incomplete data several important observations have been made about the MRSA bacteraemia occurring in patients in ERF. First, there was considerable variation in rates of MRSA blood related infections between centres in England. Several centres have reported low or zero rates. How those centres have achieved such results is not covered in this report, but shared learning between centres will be of value to further improve the national picture. It is tempting to speculate on what steps have been taken in centres that had high levels of infection in previous surveys. This may have included the adoption of the High Impact changes for renal catheters [High Impact Intervention No. 3 Renal dialysis catheter care bundle; available at www.dh.gov.uk], or the use of antibiotic catheter restricted locks [17]. Analysis of practice patterns in centres with continuing high rates will be equally instructive.

However, one factor that remains clear is the association with the use of venous catheters. MRSA blood infections within the dialysis population account for $4.2 \%$ of all bacteraemias within England. The relative risk for MRSA bacteraemia in a patient on haemodialysis is 100 fold higher than the general population, but for a patient utilizing a venous catheter that risk is 800 fold higher. Consequently, one focus to reducing the risk of infection in a vulnerable population is first to reduce the use of such catheters. If a venous catheter is required, then the risk of infection must be mitigated with meticulous care.

\section{References}

1 UK Renal Registry. The National Dialysis Access Survey: preliminary results. Chapter 6, UK Renal Registry 8th Annual Report. Bristol: UK Renal Registry, 2005.

2 Sarnak MJ, Jaber BL. Mortality caused by sepsis in patients with endstage renal disease compared with the general population. Kidney Int 2000;58(4):1758-64.

3 Kuo CB, Lin JC, Peng MY, Wang NC, Chang FY. Endocarditis: impact of methicillin-resistant Staphylococcus aureus in hemodialysis patients and community-acquired infection. J Microbiol Immunol Infect 2007;40(4):317-24.

4 Klevens RM, Edwards JR, Andrus ML, Peterson KD, Dudeck MA, Horan TC. Dialysis Surveillance Report: National Healthcare Safety
The spotlight on infection control has led to a reduction in infection within the dialysis population. Although comparison with the previous two Renal Registry surveys should be made with caution given the different methodologies, there is evidence of substantial reductions in MRSA bacteraemia. Infection remains a leading cause of mortality in the renal replacement population. If reductions in MRSA are accompanied by parallel reductions in the rates of other infections, one may anticipate survival benefits in future.

Looking forward, the data system will be improved. It will continue to link microbiological data with patient therapy, but a review of the system links should improve data completeness. Second, as part of the Kidney Care national audit [18], data from the HPA, Renal Registry and Hospital Episode Statistics (HES) will allow the linkage of bacteraemia data, hospital episodes and access, to better understand the links between infection, vascular access and morbidity and mortality for dialysis patients. Finally, the National Clinical Director for Kidney Care has established a Healthcare Associated Infections (HCAI) sub group to coordinate strategy in this area.

\section{Summary and Conclusions}

The first year of the Renal component of the HCAIDCS reporting scheme has confirmed the high rate of MRSA bacteraemia amongst patients receiving dialysis in England. Although there is evidence of an overall reduction, there is marked variability between centres in the rate of MRSA bacteraemia. The findings confirm the association of venous catheters and the risk of MRSA blood stream infections amongst patients receiving long term haemodialysis.

Conflict of interest: none

Network (NHSN)-data summary for 2006. Semin Dial 2008;21(1):24-8.

5 Katneni R, Hedayati SS. Central venous catheter-related bacteremia in chronic hemodialysis patients: epidemiology and evidence-based management. Nat Clin Pract Nephrol 2007;3(5):256-66.

6 Kamalakannan D, Pai RM, Johnson LB, Gardin JM, Saravolatz LD. Epidemiology and clinical outcomes of infective endocarditis in hemodialysis patients. Ann Thorac Surg 2007;83(6):2081-6.

7 Ishani A, Collins AJ, Herzog CA, Foley RN. Septicemia, access and cardiovascular disease in dialysis patients: the USRDS Wave 2 study. Kidney Int 2005;68(1):311-8.

8 Rayner HC, Besarab A, Brown WW, Disney A, Saito A, Pisoni RL. Vascular access results from the Dialysis Outcomes and Practice Patterns 
Study (DOPPS): performance against Kidney Disease Outcomes Quality Initiative (K/DOQI) Clinical Practice Guidelines. Am J Kidney Dis 2004;44(5 Suppl 2):22-6.

9 Inrig JK, Reed SD, Szczech LA, Engemann JJ, Friedman JY, Corey GR, et al. Relationship between clinical outcomes and vascular access type among hemodialysis patients with Staphylococcus aureus bacteremia. Clin J Am Soc Nephrol 2006;1(3):518-24.

10 Moist LM, Trpeski L, Na Y, Lok CE. Increased hemodialysis catheter use in Canada and associated mortality risk: Data from the Canadian Organ Replacement Registry 2001-2004. Clin J Am Soc Nephrol 2008;3: 1726-1732.

11 Oliver MJ, Rothwell DM, Fung K, Hux JE, Lok CE. Late creation of vascular access for hemodialysis and increased risk of sepsis. J Am Soc Nephrol 2004;15(7):1936-42.

12 George A, Tokars JI, Clutterbuck EJ, Bamford KB, Pusey C, Holmes AH. Reducing dialysis associated bacteraemia, and recommendations for surveillance in the United Kingdom: prospective study. BMJ 2006;332(7555):1435.
13 Wyllie DH, Peto TE, Crook D. MRSA bacteraemia in patients on arrival in hospital: a cohort study in Oxfordshire 1997-2003. BMJ 2005;331(7523):992.

14 Duckworth G, Charlett A. Improving surveillance of MRSA bacteraemia. BMJ 2005;331(7523):976-7.

15 Duerden B. Controlling healthcare-associated infections in the NHS. Clin Med 2008;8(2):140-142.

16 Health Protection Agency. Summary points and commentary on Quarterly (April 2006 to June 2008) and financial year (April 2001 to March 2008) MRSA bacteraemia data derived from Mandatory Surveillance, September 2008. http://www.hpa.org.uk/web/HPAwebFile/ HPAweb_C/1221638192568. London: Health Protection Agency, 2008.

17 Jaffer Y, Selby NM, Taal ;MW, Fluck RJ, McIntyre CW. A meta-analysis of hemodialysis catheter locking solutions in the prevention of catheterrelated infection. Am J Kidney Dis. 2008 Feb;51(2):233-41.

18 The Information Centre. The National Kidney Care Audit. http:// www.ic.nhs.uk/services/national-clinical-audit-support-programmencasp/kidney-care. Leeds, 2008. 